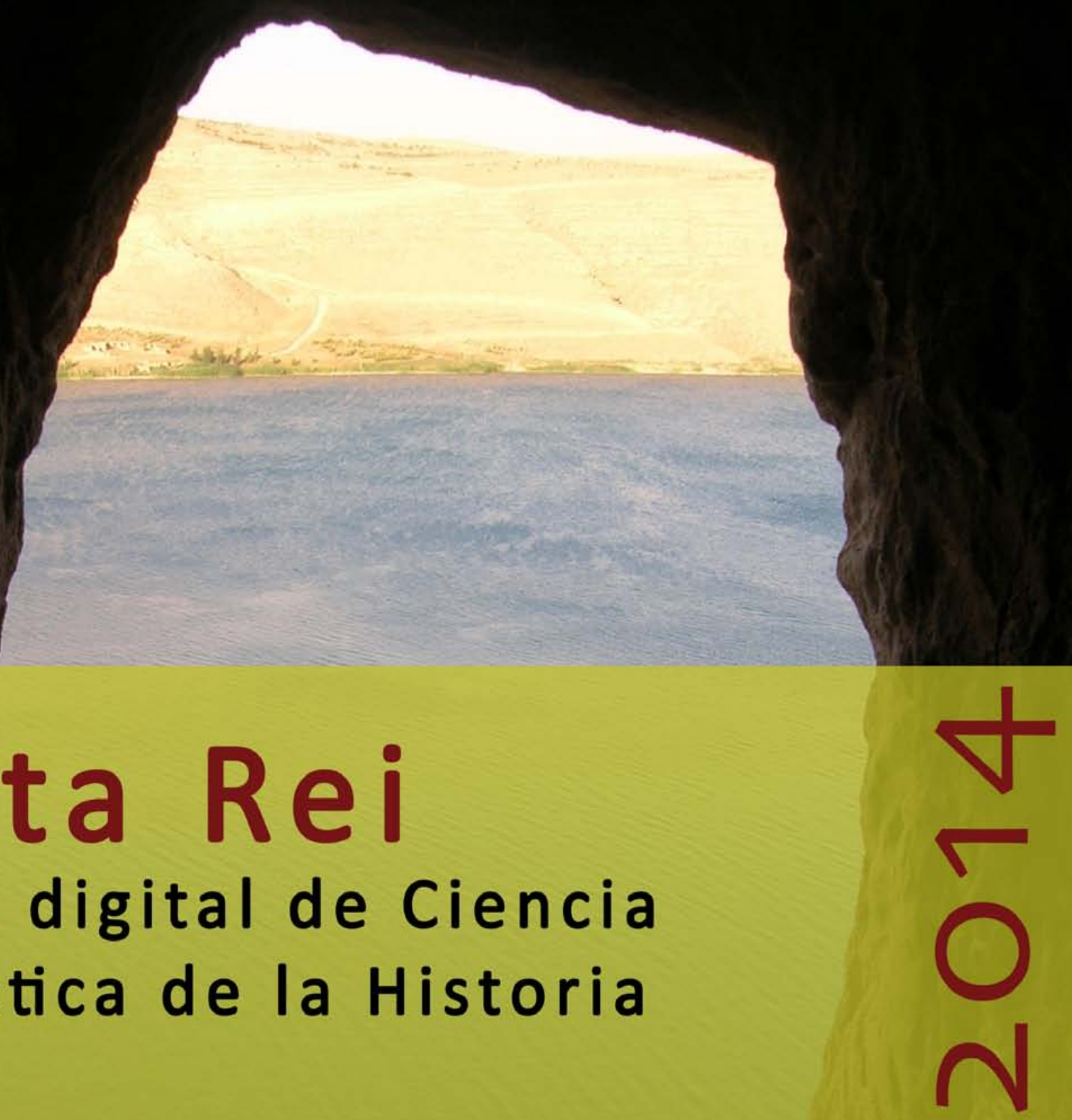





\section{Panta

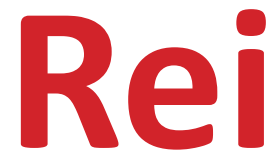 \\ Revista Digital de Ciencia \\ y Didáctica de la Historia}

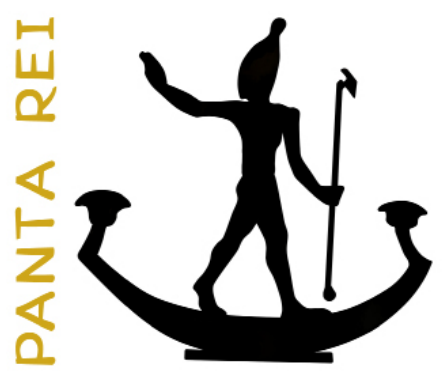

2014

Revista anual

Fecha de inicio: 1995

Revista Panta Rei. pantarei@um.es

\section{Edita:}

Centro de Estudios del Próximo Oriente y la

Antigüedad Tardía - CEPOAT

Edificio Universitario Saavedra Fajardo.

Universidad de Murcia

C/ Actor Isidoro Máiquez, 9

30007 - MURCIA - ESPAÑA

Teléfono: (+34) 868883890

cepoat@um.es

Web: www.um.es/cepoat/pantarei

Edición 2014

ISSNe: 2386-8864

ISSN: $1136-2464$

Depósito legal: MU-966-1995
cepoAt

UNIVERSIDAD DE MURCIA centro de estudios del próximo oriente y la antigüedad tardia

En Portada: Río Éufrates desde un eremitorio cristiano primitivo (Fotografía de Alejandro Egea).

Responsables de los textos:

Sus autores.

Responsable de la presente edición: Consejo Editorial Panta Rei. 


\section{CONSEJO DE REDACCIÓN}

\section{Coordinador editorial}

Egea Vivancos, Alejandro

[Didáctica de las Ciencias Sociales, UMU]

\section{Editores}

Botí Hernández, Juan Jesús

[CEPOAT, UMU]

Sáez Giménez, David Omar [CEPOAT, UMU]

\section{Secretaria}

Arias Ferrer, Laura

[Didáctica de las Ciencias Sociales, UMU]

\section{Responsable informático}

Martínez García, José Javier

[CEPOAT, UMU]

\section{Traducción y corrección lingüística}

Martínez Martínez, Cristina

[Sociedad Española de Lenguas Modernas]

\section{CONSEJO ASESOR}

Albero Muñoz, M. ${ }^{a}$ del Mar [H. ${ }^{a}$ del Arte, UMU]

Cobacho López, Ángel

[Derecho, UMU]

Egea Bruno, Pedro M. ${ }^{a}$

[Historia Contemporánea, UMU]

García Atienzar, Gabriel

[Prehistoria, UA]

González Monfort, Neus

[Didáctica de las Ciencias Sociales, UAB]

Haber Uriarte, María

[Prehistoria, UMU]

Irigoyen López, Antonio

[Historia Moderna, UMU]

Mahony, Simon

[Digital Humanities, UCL, Reino Unido]

Marsilla de Pascual, Francisco Reyes

[Técnicas historiográficas, UMU]

Miralles Maldonado, José Carlos

[Filología Clásica, UMU]

Molina Gómez, José Antonio

[Historia Antigua, UMU]

Noguera Celdrán, José Miguel

[Arqueología, UMU]

Pérez Molina, Miguel Emilio

[Filología Clásica, UMU]

Prados Martínez, Fernando

[Arqueología, UA]

Sánchez Ibáñez, Raquel

[Didáctica de las Ciencias Sociales, UMU]

Sancho Gómez, Miguel Pablo

[Educación, UCAM]

Vilar García, María José

[Historia Contemporánea, UMU] 
Presentación

Todo fluye. Renovarse y crecer

Alejandro Egea Vivancos, Laura Arias Ferrer, Juan Jesús Botí Hernández y David Omar Sáez Giménez

Artículos

La sociedad hiperbórea: ¿utopía o mito? Reflexiones acerca de la naturaleza y significado del relato hiperbóreo.

José Ángel Castillo Lozano.

Aproximación a la figura de una matrona romana culta y poderosa. El caso de Julia Domna.

Consuelo Isabel Caravaca Guerrero.

¡Arrasar la Vendée! Guerra Civil y Columnas Infernales en pleno corazón de la Revolución Francesa.

Benjamín Cutillas Victoria.....

Arte y expresión en el pensamiento de E. H. Gombrich.

Patricia Castiñeyra Fernández.

Odiseo a través de la parodia. Desmitificación e ironía de una Ítaca nostálgica en Prometeo de Pérez de Ayala y ¿Por qué corres, Ulises? de Gala.

Carmen María López López.

Análisis de la actividad didáctica del Museo de Arte Ibérico El Cigarralejo (Mula, Murcia).

Julio García Toral......

La Transición española en $4 .^{\circ}$ ESO. Un estudio de caso de los significados.

Borja Santiago Arnoso.

Entrevista

Entrevista al profesor D. Michael Walker.

Consuelo Isabel Caravaca Guerrero.

Reseñas

II Congreso de la Asociación Internacional de Investigación para la Educación de la Historia y las Ciencias Sociales.

Elvira Barriga y Rodrigo Salazar.

I Congreso de Jóvenes Investigadores del Mundo Antiguo de la Universidad de Murcia.

Pedro David Conesa Navarro

II Congreso Internacional de Educación Patrimonial.

Tània Martínez Gil.....

Ruiz-Gálvez Pliego, M. (2013). Con el fenicio en los talones. Los inicios de la Edad del Hierro en la cuenca del Mediterráneo. Barcelona: Editorial Bellaterra. 377 págs.

Celso Sánchez Mondéjar..

Normas de publicación/Publishing rules 



\title{
II Congreso de la Asociación Internacional de Investigación para la Educación de la Historia y las Ciencias Sociales
}

\author{
Para citar este artículo: Barriga, E. y Salazar, R. (2014). II Congreso de la Asociación de \\ Investigación para la Educación de la Historia y las Ciencias Sociales. Panta Rei. Revista Digital \\ de Ciencia y Didáctica de la Historia, 149-151.
}

ISSNe: 2386-8864

DOI: http://dx.doi.org/10.6018/pantarei/2014/10

El II Congreso organizado por la Asociación Internacional de Investigación para la Educación de la Historia y las Ciencias Sociales (IRAHSSE - AIRDHSS: International Research Association for History and Social Science Education) tuvo lugar el 11, 12 y 13 de setiembre de 2014 en la Universidad de Friburgo (Suiza). El encuentro congregó a personal docente e investigador de la mayor parte de universidades de Europa, Latinoamérica, Canadá y África relacionado con la educación formal y no formal, así como personal responsable de equipamientos patrimoniales, y una pequeña representación de jóvenes pre-doctorales interesados en la Didáctica de la historia y las ciencias sociales.

El congreso inicio su apertura puntualmente a las 10.00 horas del día 11 de septiembre ofreciendo una calurosa bienvenida a los asistentes por parte de Pierre-Phillipe Bugnard (Presidente de la Conferencia), Luigi Cajani (Presidente de la Asociación IRAHSSE AIRDHSS), Anne Philipona y Prisca Lehmann (coordinadoras del encuentro) y Bernadette Charlier (representante del Departamento de Ciencias de la Educación de la Universidad de Friburgo). El cierre de la presentación de bienvenida estuvo a cargo de Phillippe Joutard que deleitó al público con una formidable conferencia titulada: Guerres mondiales, historie et mémoires à l'escole en la que defendió que una educación por la paz no era posible sin una robusta enseñanza de la historia que muestre diferentes perspectivas de una realidad compleja.

La estructura del evento se desarrolló en torno a sesiones de una hora y media que recopilaban tres o cuatro paneles de comunicación temáticos que se realizaban de forma paralela. En cada panel de comunicación se presentaban y defendían tres trabajos de investigación relacionados con la educación histórica y de las ciencias sociales durante unos veinte minutos aproximadamente. Este tipo de dinámica organizativa permitió a los asistentes movilizarse por los paneles de comunicación según sus preferencias personales y profesionales. Todas las mesas de trabajo dedicaban la última media hora de su tiempo a generar un rico debate entre los comunicantes y el público asistente que era guiado por el moderador/a especialista en la materia.

El primer día estuvo compuesto por tres sesiones. El primer bloque de sesiones recogía tres paneles de comunicaciones que versaban sobre tres temáticas diferenciadas pero interrelacionadas: 1) Cuestiones políticas y el trabajo de la memoria; 2) Guerras internas 
y el trabajo de la memoria y 3) La política de educación de la histórica. En este último panel una de las aportaciones más interesantes fue la investigación de Giovanna Leone y Luigi Cajani. En este proyecto se grabó reacción de la expresión facial de 80 estudiantes mientras leían dos textos diferenciados (una primera versión fría y con pocos detalles y una segunda versión con una descripción violenta y dura) sobre los crímenes italianos durante la conquista de Etiopia (1935-1936). Los resultados mostraron que aquellos estudiantes que leyeron la versión más violenta cambiaron sus emociones respecto a la conquista nacional italiana. El objetivo de la segunda parte de este experimento es repetir el mismo procedimiento pero esta vez grabando las reacciones que causa la lectura de los libros de texto y posteriormente analizar los efectos de las principales herramientas didácticas utilizadas por el profesorado. El segundo y tercer bloque se abordaron investigaciones relacionadas con las temáticas de educación para la paz, los conflictos y la memoria. Destacó el panel de comunicaciones dedicado al racismo y la educación para el Holocausto dirigido por el Dr. Luigi Cajani. En concreto, se distinguió la investigación de Marc Van Berkel sobre las representaciones del Holocausto en los libros de historia de Holanda (1960-2010). Así como la presentación de Katalin Morgan de la Universidad de Witwatersrand (Sud África) sobre cómo responden los jóvenes alemanes frente a testimonios en formato audiovisual de supervivientes al Holocausto.

La primera sesión del segundo día estaba organizada por cuatro bloques temáticos: 1) Educación para la paz en África; 2) La imagen de la guerra; 3) Las representaciones del Colonialismo y 4) La guerra civil Española. La agrupación en dichas temáticas facilitó que se abrieran debates muy potentes después de la exposición de los comunicantes. La siguiente sesión abordaba temas más generales que tenían en cuenta las siguientes tendencias: 1) La política de la enseñanza de la historia; 2) Las prácticas y estrategias de enseñanza donde Penelope Harnett describió dos aproximaciones interactivas muy interesantes para la enseñanza de la historia en educación primaria; 3) Las representaciones y la conciencia histórica moderada por Maria do Céu de Melo y por último, Neus González moderó la mesa sobre: 4) Suiza como testigo de guerras. La tarde estuvo en dividida en dos sesiones diferenciadas: en la primera parte se destinó el tiempo a tratar diferentes intereses didácticos y de innovación educativa: 1) Las representaciones de los alumnos; 2) La primera guerra mundial y la formación de profesorado en pensamiento histórico; 3 ) Los manuales de guerra; y 4) Análisis de libros de texto. En estas últimas sesiones las investigaciones académicas de cultura lusófona (de la Universidade do Minho, Universidade do Porto y Universidade Federal do Paraná en Curitiba) obtuvieron un gran protagonismo ya que sus trabajos avanzan en la línea del aprendizaje histórico del alumnado generado a partir del proceso de enseñanza-aprendizaje de casos concretos que son llevados al aula. La investigación que Glória Solé, Diana Reis y Andreia Machado compartieron con la audiencia fue especialmente cautivadora por el juego didáctico que implicaba ponerla en práctica en el aula. Expusieron las principales conclusiones fruto del uso de las leyendas y la ficción histórica en el aprendizaje de la historia de educación primaria. La segunda parte de la tarde estuvo destinada a congregar la reunión anual de la Asamblea General de la Asociación organizadora del evento: se realizó un estado de la cuestión sobre de dónde se partía, en qué punto nos encontrábamos y hacía dónde nos dirigíamos en materia de investigación en didáctica de la historia y las ciencias sociales.

El broche final en el último día del encuentro reunió en una misma mesa redonda a Zhou Chunsheing, Paul Grossrieder, Déris Mudiandam y Hans-Ulrich Jost para charlar sobre cómo puede ayudar la escuela y el aprendizaje en el aula a que existiera una efectiva 
educación por la paz. El segundo Congreso de la Asociación Internacional en la temática de Guerra y Paz fue un espacio ideal para poner en común el trabajo realizado desde diferentes lugares del mundo. Los participantes del evento pudieron compartir preocupaciones, alternativas didácticas y reflexiones profundas con el objetivo de aumentar el conocimiento de la materia. La difusión de los resultados de todas las investigaciones presentadas tiene en común que pretenden provocar cambios en los estilos de enseñanza de las ciencias sociales. Eventos como los organizados ayudan que ese cambio cada vez esté más cerca de ser algo real.

Elvira Barriga

Universidad de Barcelona

Rodrigo Salazar

Universidad de Barcelona 



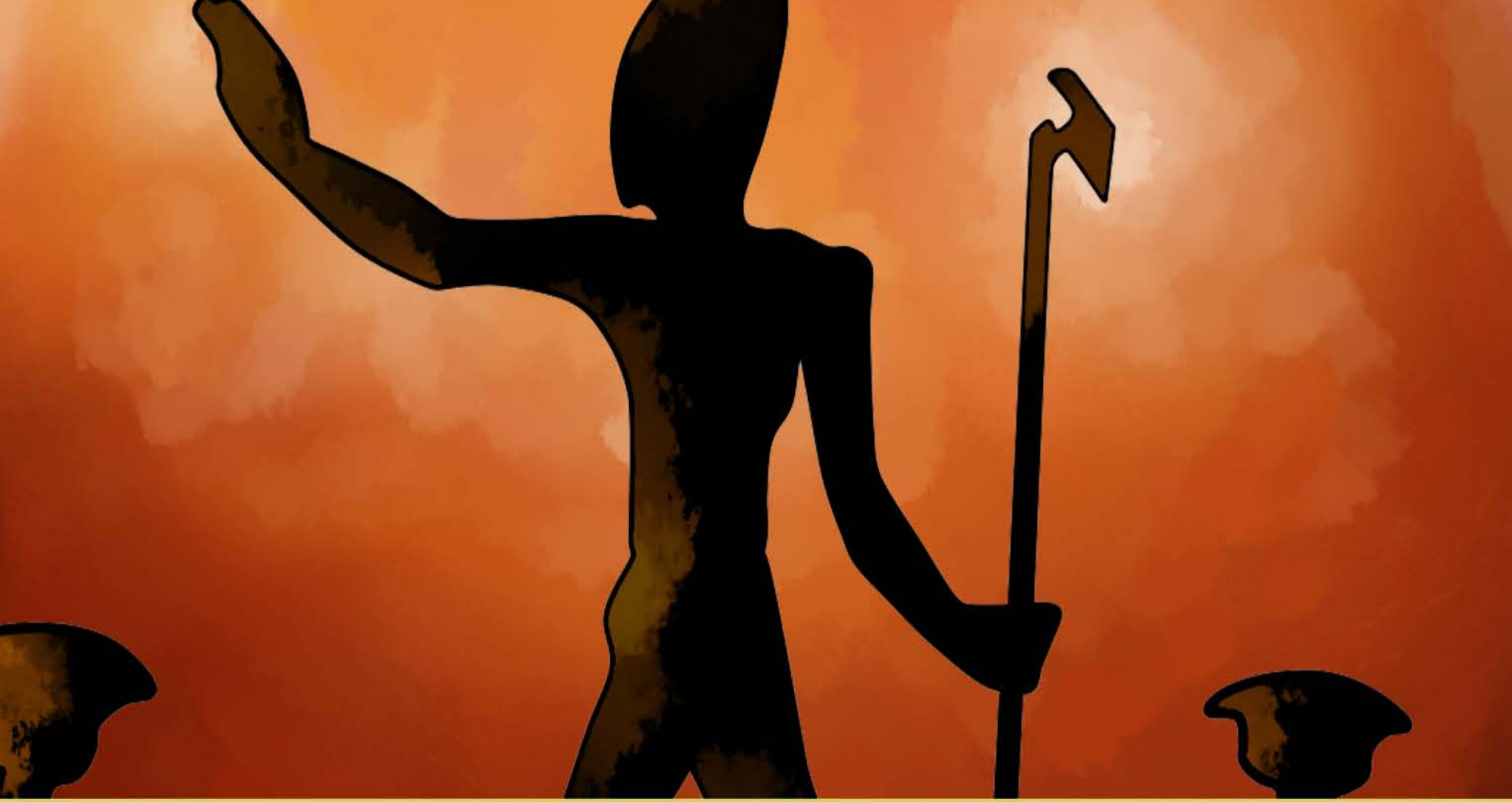

